\title{
Dosis de diálisis alcanzada en pacientes en hemodiálisis según el acceso vascular empleado
}

\author{
Raquel Pelayo Alonso, Patricia Martínez Álvarez, Ma José Cagigas Villoslada, Camino Villa Llamazares, \\ $M^{a}$ Eugenia Cuadrado Mantecón, Magdalena Gándara Revuelta \\ Unidad de Hemodiálisis. Servicio de Nefrología. Hospital Universitario Marqués de Valdecilla. Santander. España
}

\section{Introducción}

Uno de los principales objetivos que se busca en el paciente en hemodiálisis es alcanzar una diálisis adecuada. Muchos autores defienden la dosis dialítica como un marcador para una diálisis adecuada. La bibliografía refiere una menor dosis dialítica en los pacientes con catéter permanente.

Objetivos: Evaluar la dosis de diálisis alcanzada por el paciente en función de su acceso vascular.

Material y Método: Estudio descriptivo prospectivo en pacientes en programa de hemodiálisis, que se dializaron al menos 1 mes, a través del mismo acceso vascular permanente.

La dosis dialítica se valoró mediante $\mathrm{Kt}$ y Kt/V. Se recogieron datos de Kt medidos por el monitor mediante dialisancia iónica en todas las sesiones del período de estudio. El Kt se consideró adecuado si se alcanzan 4045L en mujeres ó 45-50L en hombres, y como óptimo, si cifras mayores. El Kt/V fue calculado mediante la fórmula de Daugirdas $2^{\mathrm{a}}$ generación, considerándose como adecuado si $\geq 1,3$.

Resultados: Se estudiaron 45 pacientes con edad media de $66,78 \pm 15.86$ años. El $31 \%$ se dializa a través de Fístula-Arteriovenosa (FAV). Los portadores de FAV alcanzaron Kt de 49,68L frente a 47,6L en los portadores de Catéter Venoso Central Perma-

Correspondencia:

Raquel Pelayo Alonso

Unidad de Hemodiálisis. $1^{a}$ planta Edificio 2 de Noviembre Hospital Universitario Marqués de Valdecilla Avda. Valdecilla, 25. 39008 Santander

E-mail: sanesteban6@gmail.com nente (CVC-P). El Kt/V fué 1,56 para FAV y 1,55 para CVC-P sin que estas diferencias fueran estadísticamente significativas. El Kt/V fue adecuado en el $78,6 \%$ de los portadores de FAV y el $77,4 \%$ de los portadores de CVC.

Conclusión: Según nuestros datos, no existe diferencia en la dosis de diálisis alcanzada según el acceso vascular empleado.

PALABRAS CLAVE: dosis de diálisis; hemodiálisis; acceso vascular; diálisis adecuada.

Reached Dialysis dose in hemodialysis patients according to the type of vascular access

\section{Abstract}

Introduction: One of the main objectives of the haemodialysis patient is to achieve adequate dialysis. Many authors defend the dialysis dose as a marker for adequate dialysis. The literature refers to a lower dialysis dose in patients with a permanent catheter.

Objective: To evaluate the reached dialysis dose by the patient according to the type of vascular access.

Material and Method: Prospective descriptive study in patients in haemodialysis program, who were dialyzed at least 1 month, through the same permanent vascular access.

The dialysis dose was assessed by $\mathrm{Kt}$ and $\mathrm{Kt} / \mathrm{V}$. Kt data, measured by the monitor, were collected by ionic dialysance in all sessions of the study period. Kt was considered adequate if 40-45 $\mathrm{L}$ were reached in women or 45-50L in men, and as an optimum, if larger 
numbers were reached. The $\mathrm{Kt} / \mathrm{V}$ was calculated using the Daugirdas' 2nd generation formula, considering it adequate if $\geq 1.3$.

Results: Forty-five patients with a mean age of $66.78 \pm$ 15.86 years were studied. Arteriovenous Fistula (AVF) was used in $31 \%$ of haemodialysis seasons. Patients with AVF reached a $\mathrm{Kt}$ of $49.68 \mathrm{~L}$ versus $47.6 \mathrm{~L}$ of those with Permanent Central Venous Catheter (P-CVC). $\mathrm{Kt} / \mathrm{V}$ was 1.56 for patients with AVF and 1.55 for those with $\mathrm{P}-\mathrm{CVC}$, although without statistically significant differences. $\mathrm{Kt} / \mathrm{V}$ was adequate in $78.6 \%$ of those with AVF and $77.4 \%$ of patients with P-CVC.

Conclusions: According to our data, there is no difference in the reached dialysis dose according to the type of vascular access employed.

KEYWORDS: dialysis dose; haemodialysis; vascular access; adequate dialysis.

\section{Introducción}

Uno de los principales objetivos que se busca en el paciente en hemodiálisis (HD) es alcanzar una diálisis adecuada, es decir, un tratamiento sustitutivo eficaz, suficiente, con buena tolerancia, que mejore la calidad de vida del paciente y prolongue su supervivencia ${ }^{1}$. Muchos autores defienden la dosis dialítica (DD) como un marcador para una diálisis adecuada, ya que se relaciona con una mejora del estado anémico, estado nutricional, control tensional y sobre la supervivencia del paciente. La DD puede ser un indicador fiable sobre el pronóstico de los pacientes hemodializados ${ }^{2-6}$. Existen diferentes métodos cuantitativos siendo el parámetro más utilizado el Kt/V para la determinación de la DD. Las guías de práctica clínica tanto nacionales como internacionales ${ }^{7}$ sugieren el indicador del Kt/V de 1.3 como un parámetro dialítico optimo; siendo recomendable aumentar este valor en el género femenino, pacientes con un peso corporal inferior a $50 \mathrm{~kg}$ y en diabéticos ${ }^{3}$.

Existen múltiples factores que intervienen en la eficacia dialítica de cada sesión de HD, de ahí que se hayan desarrollado sistemas que permiten conocer la dosis de diálisis que recibe el paciente en tiempo real, mediante la dialisancia iónica efectiva. Por esta razón, en los últimos años, se emplea el Kt como marcador de DD. Lowri y cols ${ }^{8}$ proponen un Kt mínimo de 40-45L para las mujeres y de 45-50L en el caso de los hombres, como indicador de DD adecuada.
Otro factor que también influye en la supervivencia de estos pacientes es la presencia de un acceso vascular (AV) adecuado, siendo uno de los factores más condicionantes sobre el la calidad del tratamiento de HD'. De los tres tipos de AV existentes en la actualidad, la fístula arteriovenosa (FAV) es la primera elección. La guías de la Sociedad Española de Nefrología (SEN) considera como un indicador de calidad el porcentaje de pacientes portadores de FAV, para unidades de HD que tengan cifras superiores del $80 \%$ de FAV y un $10 \%$ catéteres venosos centrales permanentes (CVC-P $)^{10}$. Sin embargo, en los últimos años, debido al cambio en el perfil del paciente incluido en programas de HD, como principal factor influyente, aumentando las tasas de CVC-P9. Según datos del informe anual del estudio DOPPS del año 2012, en España, el 66,6\% de los pacientes era portador de FAV frente a un $24,9 \%$ CVC-P ${ }^{11}$. La bibliografía evidencia una menor DD conseguida en los pacientes con CVC-P. ${ }^{12}$ En los últimos años, hemos observado en nuestra unidad un aumento en el porcentaje de CVC-P, siendo uno de los motivantes para plantear el presente estudio.

El objetivo de este estudio fue evaluar la dosis de diálisis alcanzada por el paciente en función de su acceso vascular así como otros posibles factores que puedan influir en la eficacia dialítica.

\section{Material y Método}

Se realizó un estudio observacional descriptivo prospectivo en 45 pacientes de la unidad de hemodiálisis del Hospital Marqués de Valdecilla, Santander. Se incluyeron todos los pacientes incluidos en programa de HD crónica con un tiempo de uso de al menos 1 mes y utilizando el mismo AV durante todo el periodo de estudio y que consintieron participar una vez recibida la información pertinente. Se excluyeron aquellos pacientes que por si situación clínica, no realizaban una pauta fija de HD. El período de estudio fue de 4 semanas.

La DD se valoró mediante $\mathrm{Kt}$ y $\mathrm{Kt} / \mathrm{V}$. Se recogieron datos de Kt medidos por el monitor a través de dialisancia iónica en todas las sesiones del período de estudio, una vez finalizada la sesión. El Kt/V fue valorado el día central de la primera y la última semana de estudio al igual que la tasa de recirculación. Para el cálculo del Kt/V se empleó la fórmula de Daugirdas $2^{\mathrm{a}}$ generación $^{13}$. 


\section{$\mathrm{Kt} / \mathrm{V}=-\mathrm{Ln}[(\mathrm{C} 2 / \mathrm{C} 1)-(0,008 \mathrm{xT})]+[4-3,5 x(\mathrm{C} 2 / \mathrm{c} 1)] x U F / P e s 0$}

Donde $\mathrm{C} 1$ y $\mathrm{C} 2$ son los niveles de urea inicial y final; $\mathrm{T}$ es el tiempo de hemodiálisis en horas y UF es la diferencia de peso antes y después de la HD en kilogramos. La muestra de urea inicial se obtuvo antes de comenzar la sesión, directamente del acceso vascular del paciente y la muestra final, al finalizar la sesión de HD, y tras mantener el flujo sanguíneo a $50 \mathrm{ml} / \mathrm{min}$ durante 2 minutos, extrayendo la muestra sanguínea del botón arterial del circuito extracorpóreo, según el protocolo de nuestra Unidad.

Para el cálculo de la tasa de recirculación, se utilizó la fórmula:

\section{\% recirculación= (Urea periférica-Urea arterial)/ (Urea periférica/Urea venosa) x100}

La obtención de muestras se produjo una vez alcanzado el flujo sanguíneo deseado, extrayendo una muestra del botón arterial y otra muestra del botón venoso, de forma simultánea para obtener los valores de urea arterial y venosa. Para obtener el valor de urea periférica, se obtuvo una tercera muestra, del botón arterial tras mantener el flujo sanguíneo a $50 \mathrm{ml} / \mathrm{min}$ durante 30 segundos, según protocolo de la Unidad.

No se modificaron otros parámetros que pudiesen influir en la DD durante el periodo de estudio, como la duración de la sesión, el dializador o la técnica dialítica. Se recogieron datos sociodemográficos y clínicos de la Historia Clínica del paciente, así como parámetros relativos al tratamiento dialítico (AV, dializador, tipo de HD, duración y flujo sanguíneo). Se establecieron 3 grupos de pacientes, en función de los resultados de KT: Kt optimo (mujeres $>45 \mathrm{~L}$; hombres $>50 \mathrm{~L}$ ); Kt normal (mujeres $40-45 \mathrm{~L}$; hombres 45-50 L); Kt bajo (mujeres $<40 \mathrm{~L}$; hombres $<45 \mathrm{~L}$ ); y otros 2 grupos según el $\mathrm{Kt} / \mathrm{V}$ alcanzado: adecuado ( $\geq 1.3$ ) inadecuado $(<1.3)$.

El análisis estadístico se realiza con el programa SPSS versión 15.0 para Windows. Se realizó un análisis descriptivo de las variables a estudio, expresándose las variables cuantitativas como media, desviación estándar o mediana y las cualitativas como frecuencia y porcentaje. El contraste de hipótesis para variables cuantitativas se realizó mediante $t$ de Student y análisis de varianza (ANOVA) y chi-cuadrado para las variables cualitativas. Se consideró como significación estadística una $p<0.05$.

\section{Resultados}

Se analizaron 45 pacientes, con una edad media de $66,78 \pm 15.86$ años, siendo un $60 \%$ hombres. El $31 \%$ de la muestra se dializaba a través de FAV y el resto, mediante un CVC-P. En la Tabla 1 se indican las características clínicas y parámetros dialíticos, en función del AV empleado.

En cuanto a la DD (Tabla 2), los portadores de FAV alcanzaron un $\mathrm{KT}$ de $49.68 \mathrm{~L}$ frente a $47.6 \mathrm{~L}$ para los portadores de CVC-P; así como un Kt/V de 1.56 y 1.55 respectivamente, no observándose diferencias estadísticamente significativas. No hubo diferencias estadísticamente significativas entre la DD (medida por $\mathrm{Kt} 0 \mathrm{Kt} / \mathrm{V}$ ) relacionado con la técnica de diálisis 0 el tipo de dializador. La distribución de los pacientes en función del tipo de Kt alcanzado, se muestra en el Gráfico 1 y la adecuación del Kt/V en función del AV, se representa en el Gráfico 2.

Tabla 1. Características clínicas y parámetros dialíticos según el acceso vascular del paciente.

\begin{tabular}{|c|c|c|c|}
\hline & & FAV & CVC-P \\
\hline Sexo & $\begin{array}{l}\text { Mujer } \\
\text { Hombre }\end{array}$ & $\begin{array}{l}13,3 \% \\
17,8 \%\end{array}$ & $\begin{array}{l}26,7 \% \\
42,2 \%\end{array}$ \\
\hline Edad & & $65 \pm 23$ años & $68 \pm 12$ años \\
\hline Nefropatía & $\begin{array}{l}\text { Glomerular } \\
\text { Diabética } \\
\text { Vascular } \\
\text { Instersticial } \\
\text { Otra }\end{array}$ & $\begin{array}{c}2,2 \% \\
2,2 \% \\
13,3 \% \\
4,4 \% \\
8,9 \%\end{array}$ & $\begin{array}{r}6,7 \% \\
17,8 \% \\
15,5 \% \\
11,1 \% \\
17,8 \% \\
\end{array}$ \\
\hline $\begin{array}{l}\text { Técnica de } \\
\text { Hemodiálisis }\end{array}$ & $\begin{array}{l}\text { HD } \\
\text { convencional } \\
\text { HDF } \\
\text { HFR }\end{array}$ & $\begin{array}{c}6,7 \% \\
17,8 \% \\
6,7 \% \\
\end{array}$ & $\begin{array}{l}22,2 \% \\
35,5 \% \\
11,1 \% \\
\end{array}$ \\
\hline Tipo de dializador & $\begin{array}{l}\text { Policarbonato } \\
\text { Polisulfona } \\
\text { Polifenileno }\end{array}$ & $\begin{array}{c}6,7 \% \\
17,8 \% \\
6,7 \% \\
\end{array}$ & $\begin{array}{c}17,8 \% \\
40 \% \\
11,1 \% \\
\end{array}$ \\
\hline Duración HD & & $234 \pm 17$ minutos & $226 \pm 41$ minutos \\
\hline Tiempo en HD & & $66 \pm 53$ meses & $44 \pm 63$ meses \\
\hline Tiempo de uso del AV & & $59 \pm 51$ meses & $29 \pm 44$ meses \\
\hline
\end{tabular}

CVC-P: catéter permanente; HD: hemodiálisis; HDF: hemodiafiltración; HFR: hemodiafiltración con regeneración de ultrafiltrado; AV: acceso vascular). Los datos se presentan en $\%$ o como media $\pm D E$. 
Tabla 2. Características clínicas y parámetros dialíticos según el acceso vascular del paciente.

\begin{tabular}{|c|c|c|c|c|c|}
\hline & & \multicolumn{2}{|l|}{ Kt (L) } & \multicolumn{2}{|l|}{$\mathrm{Kt} / \mathrm{V}$} \\
\hline & & Media $\pm D E$ & Mediana & Media $\pm D E$ & Mediana \\
\hline \multirow[t]{2}{*}{ Acceso Vascular } & FAV & $48,76 \pm 7,53$ & 49,68 & $1,56 \pm 0,33$ & 1,51 \\
\hline & CVC-P & $46,03 \pm 6,44$ & 47,60 & $1,55 \pm 0,35$ & 1,56 \\
\hline \multirow[t]{2}{*}{ Sexo } & Hombre & $46,04 \pm 5,7$ & 46,36 & $1,66 \pm 0,31$ & 1,66 \\
\hline & Mujer & $47,43 \pm 7,53$ & 47,85 & $1,48 \pm 0,34$ & 1,5 \\
\hline \multirow[t]{2}{*}{ Técnica de HD } & HD convencional & $43,66 \pm 4,55$ & 42,83 & $1,36 \pm 0,21$ & 1,41 \\
\hline & HDF & $48,18 \pm 1,63$ & 48,85 & $1,63 \pm 0,35$ & 1,66 \\
\hline \multirow[t]{3}{*}{ Dializador } & Policarbonato & $42,39 \pm 5,5$ & 42,28 & $1,34 \pm 0,31$ & 1,38 \\
\hline & Polisulfona & $48,40 \pm 6,40$ & 48,37 & $1,69 \pm 0,31$ & 1,75 \\
\hline & Polifenileno & $48,077,9$ & 48,67 & $1,39 \pm 0,24$ & 1,39 \\
\hline
\end{tabular}

CVC-P: catéter permanente; HD: hemodiálisis; HDF: hemodiafiltración; HFR: hemodiafiltración con regeneración de ultrafiltrado; AV: acceso vascular). Los datos se expresan como media \pm desviación estándar y como mediana.

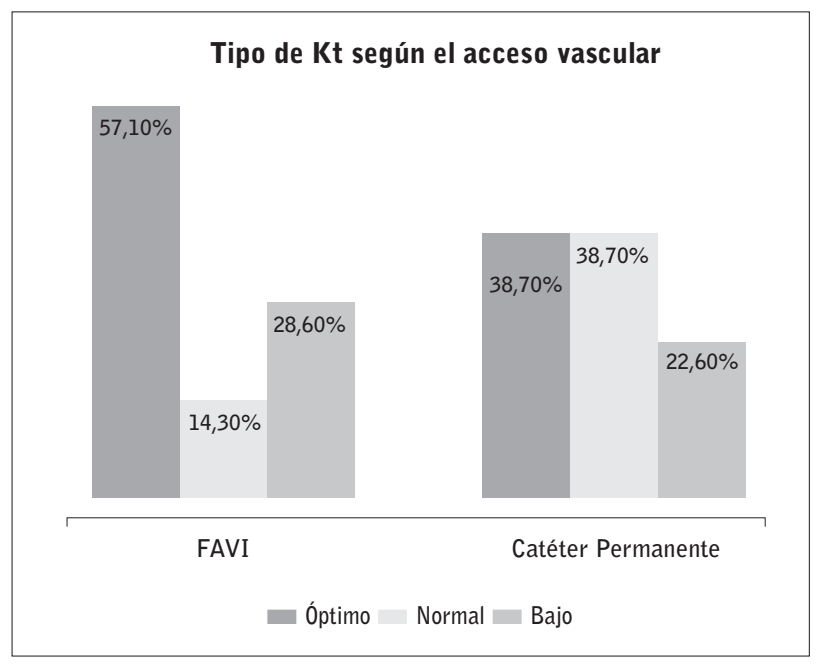

Gráfico 1. Distribución de los pacientes según el tipo de Kt alcanzado en función del acceso vascular empleado.

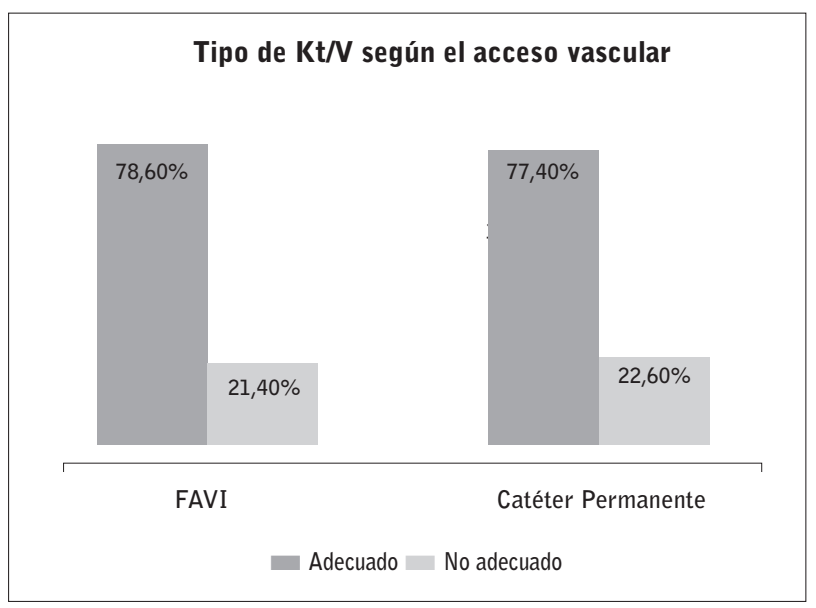

Gráfico 2. Distribución de los pacientes según el tipo de $\mathrm{Kt} / \mathrm{V}$ alcanzado en función del acceso vascular empleado.
El $57,12 \%$ de los pacientes dializados mediante FAV consiguieron un Kt óptimo frente al 38,7\% CVC-P $(p=0,063)$. Presentaron un $\mathrm{Kt} / \mathrm{V}$ adecuado el $78,6 \%$ de los portadores de FAV y el $77,4 \%$ de los portadores de CVC-P.

La tasa de recirculación para las FAVs fue de 2,41\% y para los CVC-P de 6,24\% ( $p=0,048)$. En cuanto al flujo sanguíneo, se alcanzó una mediana de flujo de 371 $\mathrm{ml} / \mathrm{min}$ con las FAVs frente a una mediana de flujo de $358 \mathrm{ml} / \mathrm{min}$ con CVC-P $(p=0,037)$.

\section{Discusión}

La medición de la dosis dialítica del paciente en tratamiento crónico de HD es de vital importancia para la repercusión futura sobre su supervivencia. Los avances tecnológicos han dotado a los monitores actuales de biosensores, que nos permiten conocer la DD en cada momento y sin necesidad de extracción de muestras sanguíneas ni pruebas de laboratorio. Esto permite poder actuar en tiempo real, y con disminución de costes, sobre aquellos factores que pueden influir en la DD, con el fin de alcanzar los estándares de calidad. ${ }^{4}, 5$ En un estudio multicéntrico ${ }^{14}$ sobre 2516 pacientes pertenecientes a 28 unidades de HD de España, en el año 2007, se demostró que ningún centro cumplía con el objetivo de alcanzar un $80 \%$ de pacientes dializados a través de FAV.

Una posible causa podría ser la situación clínica del enfermo renal al que se atiende en las unidades, de mayor edad, con mayor prevalencia de diabetes o patologías cardiovasculares que condicionan un árbol vascular 
desfavorable para la construcción o mantenimiento de una $\mathrm{AV}$ normofuncionante ${ }^{9}$. Esta situación se reproduce en nuestra población de estudio, ya que la edad media de los pacientes es elevada y presentan elevada comorbilidad, lo que se traduce en que únicamente, el 31\% de la muestra se dializa a través de FAV.

Existen numerosas publicaciones que demuestran una mayor DD alcanzada en aquellos pacientes dializados a través de $F A V^{2,4,15,16}$. Sin embargo, en nuestra muestra, la diferencia entre el Kt alcanzado con FAV y el conseguido con CVC-P apenas es de $2 \mathrm{~L}$, mientras que en el caso del $\mathrm{Kt} / \mathrm{V}$, los datos son similares, sin que exista significación estadística.

Las recomendaciones actuales de DD son de $\mathrm{Kt} / \mathrm{V}$ igual o superior a $1.3^{3,6}$ en un porcentaje al menos del $80 \%{ }^{6}$ y de un Kt mínimo de 40-45L para las mujeres y 45$50 \mathrm{~L}$ para los varones. Diferentes estudios demuestran que los pacientes dializados a través de CVC-P tienen mayor dificultades para alcanzar dosis mínimas de $\mathrm{Kt}^{4,12}$. Maduell y cols ${ }^{3}$ estiman necesario un aumento del tiempo de diálisis para conseguir un Kt mínimo de 30 minutos cuando el CVC-P se usa con los ramales en posición normal y 60 minutos si los ramales son invertidos. En nuestra muestra, no se consigue un Kt normal en el $22,6 \%$ de los dializados por CVC-P y en el $28,6 \%$ de los dializados por FAV. Sin embargo, en nuestro estudio no se ha determinado el tiempo que sería necesario aumentar la duración de las sesiones para alcanzar un Kt mínimo.

Existen numerosas alternativas para aumentar la DD. Destacan, el uso de dializadores con mayor superficie, aumentar el flujo sanguíneo o del líquido de diálisis, o añadir transporte convectivo a la técnica ${ }^{1}$. Molina y cols $^{6}$ consiguen elevar el porcentaje de pacientes que alcanzan un Kt adecuado del 36,4\% al 61,1\% aumentando el número de pacientes en tratamiento con hemodiafiltración en línea o aumentando el número de pacientes con dializadores de mayor superficie. Este comportamiento también se ve en nuestra muestra, ya que la DD es más alta cuando se realiza técnica convectiva, alcanzándose un Kt de $48 \mathrm{~L}$ y un $\mathrm{Kt} / \mathrm{V}$ de 1.66 frente a un Kt de 43L y un Kt/V de 1.36 en caso de realizar HD convencional.

Otra opción para aumentar la DD sin duda es conseguir un flujo de sangre adecuado. En un estudio previo ${ }^{12}$, se analizan las diferentes DD alcanzadas con diferentes opciones durante la diálisis, invirtiendo o no los ramales del CVC-P manteniendo el mayor flujo sanguíneo posible, o empleando diferentes flujos sanguíneos en caso de dializarse a través de una FAV. Los valores de Kt aumentan según el flujo sanguíneo alcanzado es mayor.

Pese a que la bibliografía indica que las HD con CVC-P presentan una eficacia dialítica reducida respecto a las realizadas con $\mathrm{FAV}^{6,12}$, siendo necesario aumentar en muchos casos el tiempo de diálisis, cuando el flujo sanguíneo es adecuado, es posible alcanzar los objetivos pautados. Ese comportamiento, se puede ver en nuestra muestra, ya que el flujo medio conseguido con los CVC-P es de $358 \mathrm{ml} / \mathrm{min}$ frente a los $371 \mathrm{ml} / \mathrm{min}$ alcanzados por las FAVs, lo que puede explicar los valores adecuados de DD independientemente de cual sea el AV utilizado, así como la mínima diferencia entre ambos AV. En nuestro caso, no se aumentó el tiempo de diálisis para conseguir mejorar los datos dialíticos.

Sin duda, lo más recomendable para aumentar la DD sería individualizar la pauta de diálisis en relación a aquellos factores identificados como clave en la DD: flujo sanguíneo, tiempo efectivo, dializador de mayor superficie y técnica dialítica.

Como limitación principal del estudio, destacamos el tamaño muestral y el diseño realizado del estudio, los cuales no nos permitir extrapolar los datos obtenidos. Podría ser interesante para futuras investigaciones, un mayor tamaño muestral mediante la colaboración institucional para la realización de estudios multicéntricos, en los que se tengan factores influyentes, como el flujo del líquido de diálisis.

A la vista de nuestros resultados podemos concluir que no existen diferencias en la DD alcanzada según el AV empleado. En base a datos de Kt, el porcentaje de pacientes infradializados es mayor en los que se dializan a través de FAV que en los portadores de CVC-P.

Pese al alto porcentaje de pacientes dializados a través de CVC-P de nuestra muestra, se está muy cerca de alcanzar el estándar de calidad del $80 \%$ de pacientes con $\mathrm{Kt} / \mathrm{v}$ adecuado.

\section{El autor declara que no hay conflicto de interés.}

$$
\begin{gathered}
\text { Recibido: } 8 \text { junio } 2017 \\
\text { Revisado: } 21 \text { junio } 2017 \\
\text { Modificado: } 30 \text { junio } 2017 \\
\text { Aceptado: } 5 \text { agosto } 2017
\end{gathered}
$$




\section{Bibliografía}

1. Maduell F, Arias M. Dosis de diálisis. Nefrología 2012; 7(1):447-457.

2. Gordillo R, Fernández $B$, Aller $C$, Coca $A$, Vásquez $D$, Sanz $S$, et al. Relación entre acceso vascular y dosis de diálisis en una unidad de hemodiálisis. XXXIV Congreso Anual de la Sociedad Española de Diálisis y Trasplante. [Consultado 28 marzo 2016]. Disponible en: http://www.elsevier.es/es-revistadialisis-trasplante-275-pdf-90128837-S300.

3. Maduell F, García M, Alcázar R. Dosificación y adecuación del tratamiento dialítico. Guías de centros de hemodiálisis. Nefrología 2006; 26(supl 8):15-21.

4. Fernández $A V$, Soto $S$, Arenas $M$, Sáez N, García $M^{a} \mathrm{M}$, Ortega P. Estudio comparativo de la dosis de diálisis medida por dialisancia iónica (kt) y por Kt/V. Rev Esp Enferm Nefrol 2009; 12(2):97-102.

5. Caballero R, Carrasco S, Diago A, García R, Hidalgo $\mathrm{N}$, Moreno $\mathrm{L}$, et al. Valoración de la dosis de diálisis mediante dialisancia iónica. Rev Esp Enferm Nefrol 2015; 18(1):19-22.

6. Molina $M$, Roca $S$, Alarcón RM, García MA, Jimeno C, Álvarez GM, et al. Cálculo del Kt como indicador de calidad en el área de adecuación en hemodiálisis. Nefrología 2010; 30(3):331-336.

7. Daugirdas J, Depner T. KDOQUI clinical practice guideline for hemodialysis adequacy: 2015 update. Am J Kidney Dis 2015; 66(5): 884-930.

8. Lowrie EG, Cherton GM, Lew NL, Lazarus JM, Owen WF. The urea product (Kt) as an outcomebased measure of hemodialysis dose. Kidney Int 1999; 56: 729-737.

9. Roca R. El acceso vascular para hemodiálisis: la asignatura pendiente. Nefrología 2010; 30(3):280287.
10. Rodríguez JA, González e, Gutiérrez JM et al. Guías de acceso vascular en hemodiálisis (Guías SEN). Nefrología 2005; 35(Supl 1): 3-97.

11. Cruz JM, Piera L, Fort L, Sequera P. Dialysis Outcomes and Practice Patterns Study (DOPPS) 2012. Distribución del acceso vascular en España. [Consultado 28 de marzo de 2016]. Disponible en: http://www.dopps.org/annualreport.

12. Maduell $F$, Vera $M$, Arias $M$, Fontseré $N$, Blasco $M$, Serra $N$, et al. ¿Cuánto tiempo es necesario aumentar la prescripción de hemodiálisis con la utilización de catéteres? Nefrología 2008; 28(6):633-636.

13. Maduell F, Arias M. Dosis de Diálisis. En: Lorenzo V, López JM, Martín de Francisco AL, Hernández D. Nefrología al día. Madrid: Grupo Editorial Nefrología de la Sociedad Española de Nefrología.; 2010.p.447-457.

14. Alcázar JM, Arenas MD, Álvarez-Mude F, Virto R, Maduell F, Fernández-Crespo $P$, et al. Resultados del proyecto de mejora de la calidad de la asistencia en hemodiálisis: estudio multicéntrico de indicadores de calidad de la Sociedad Española de Nefrología. Nefrología 2008; 28(6):5597-606.

15. Mutevelic A, Spanja I, Sultic-Lavic I, Koric A. The impact of vascular acceso on the adequacy of dialysis and the outcome of the dialysis treatment: one center experience. Mater Sociomed 2015; 27(2):114-117.

16. Dounosi E, Balafa 0, Gouva C, Karassavidou D, Mitsis M, Pappas E, et al. Patient clearance time with different vascular access types. Int $\mathrm{J}$ Artif Orgnas 2013; 36(12):853-860.

Este artículo se distribuye bajo una Licencia Creative Commons Atribución-NoComercial 4.0 Internacional. https://creativecommons.org/licenses/by-nc/4.0/ 